\title{
The amplification of a weak magnetic field by turbulent motion of a fluid of large conductivity
}

\author{
By P. G. SAFFMAN \\ Department of Mathematics, King's College, London
}

(Received 6 June 1963)

This paper considers the question of how large the magnetic energy can be in stationary homogeneous turbulence at large Reynolds number of an incompressible conducting fluid for which the magnetic diffusivity $\lambda$ is much less than the kinematic viscosity $v$. An approximate equation, that takes account of the effect of the Lorentz forces on the turbulence, is proposed for the spectrum function of the magnetic energy for wave-numbers lying in the equilibrium range. This equation is used to determine the magnetic spectrum function and the level of magnetic energy for the case when a statistically steady magnetic field is maintained by a relatively small input of magnetic energy, by a weak applied field say, on the scale of the energy-containing eddies; it being supposed as suggested by earlier work that the magnetic energy would eventually die away in the absence of external electromotive forces. The results are complicated, there being essentially four different régimes depending in a fairly involved way on the relative values of $\lambda / \nu$, the turbulent Reynolds number, and the ratio of the energy of the applied field to the energy of the turbulence. The main conclusions about the amplification factor are shown diagrammatically in figure 4 . The wavenumber at which the magnetic spectrum has its maximum tends to decrease, as the applied field is increased, from the conduction cut-off wave-number $\left(\epsilon / \nu \lambda^{2}\right)^{\frac{1}{4}}$ to values lying in the inertial subrange, much less than $\left(\epsilon / \nu^{3}\right)^{\frac{1}{4}}$.

The case of a turbulent dynamo is also examined, and it is concluded that, if it exists, the equilibrium magnetic energy would be given by Batchelor's criterion of equipartition of energy between the magnetic field and the small, energydissipating eddies. The magnetic energy in the dynamo is found to lie mainly in Fourier components of wave-number about $\left(\epsilon / \nu \lambda^{2}\right)^{\frac{1}{4}}$.

\section{Introduction}

It is generally accepted that the magnetic energy of a weak seed magnetic field in an electrically conducting fluid of large conductivity is greatly amplified if the fluid is in turbulent motion, but the amount by which it is amplified or the level of the magnetic energy if a statistically steady state is attained are subjects of controversy for the case when the magnetic diffusivity $\lambda$ is small compared with the kinematic viscosity $\nu$. Three main possibilities have been put forward so far. (i) The magnetic energy grows until checked by Lorentz forces when there is equipartition of energy with the small-scale components of the turbulence (Batchelor 1950). (ii) The magnetic energy continues to increase after equipartition with the small-scale kinetic energy is reached, and equipartition 
reaches progressively greater length-scales as time passes until there is equipartition with the total kinetic energy of the turbulence (Bierman \& Schliter 1950). (iii) The growth of magnetic energy, if there is no back-reaction on the turbulence, is limited by Ohmic dissipation; and in the absence of a supply of magnetic energy provided by externally applied electromotive forces, the magnetic energy eventually decays to zero (Saffman 1963, hereafter denoted by S). Although possibilities (i) and (ii) are mutually exclusive, possibility (iii) clearly does not exclude (i) or (ii), for the action of the Lorentz forces on the eddies generating magnetic energy may become dominant and stop the growth before Ohmic dissipation is significant, as clearly must be the case if $\lambda=0$ exactly. However, the contention in (iii) that the magnetic energy eventually decays to zero in the absence of external sources is not consistent with the existence of a turbulent dynamo, as opposed to amplifier, whose strength is given by possibilities (i) or (ii).

In the present work, this problem will be examined with the aid of intuitive hypotheses about the interaction of the turbulence and the magnetic field that are suggested by the analysis in S. In that paper, an equation for the magnetic energy spectrum function was derived for wave-numbers $k$ greater than the Kolmogorov wave-number $k_{d}=\left(\epsilon / \nu^{3}\right)^{\frac{1}{4}}$ ( $\epsilon=$ rate of turbulent energy dissipation per unit mass), and this equation will be extended in a plausible and consistent manner to hold throughout the equilibrium range of wave-numbers and also to take account of the effect of the Lorentz forces on the turbulence. An expression for the magnetic spectrum function is then deduced, which may be used to estimate the amplification of magnetic energy by turbulence.

It will be supposed throughout that the turbulence is statistically stationary and isotropic, being maintained against viscous dissipation by external mechanical forces. The magnetic field will also be assumed isotropic. Insofar as we shall concentrate on the spectrum in the equilibrium range, these assumptions are not unduly restrictive if the turbulent Reynolds number is sufficiently large. The fluid will be taken as incompressible with uniform physical properties. It should perhaps be stressed that the problem being considered is the generation of magnetic energy by homogeneous turbulence; the effect of large-scale inhomogeneities in either the fluid motion or the magnetic field, which may produce magnetic energy on a large scale, are outside the scope of the present discussion.

The magnetic spectrum function for the case of small magnetic Prandtl number but large magnetic Reynolds number has been investigated by Moffatt (1961) with the assumption that Ohmic dissipation sets the level of magnetic energy.

\section{The equation for the magnetic energy spectrum function}

We consider as a preliminary the spectrum function $\Gamma_{\theta}(k)$ of a conserved scalar quantity $\theta$ (e.g. temperature) convected by the turbulent fluid and subject to a molecular diffusivity $\lambda$ that is much less than $\nu$. The form of the spectrum for wave-numbers somewhat greater than $k_{d}$ was analysed by Batchelor (1959), and it was pointed out in $\mathrm{S}$ that the results imply that $\Gamma_{\theta}$ satisfies for $k \gg k_{d}$

$$
\frac{\partial \Gamma_{\theta}}{\partial t}+2 \lambda k^{2} \Gamma_{\theta}=\frac{\partial}{\partial k}\left(\gamma k \Gamma_{\theta}\right)
$$


where $\gamma(<0)$ is a measure of the rate at which material surfaces are brought together by the straining motion associated with the small eddies, and its value is probably not very different from $-0.5(\epsilon / v)^{\frac{1}{2}}$.

In the inertial subrange of wave-numbers $L^{-1} \ll k \ll k_{d}(L=$ length characteristic of the energy containing eddies), the concept of a cascade of spectral density leads to the approximate expression $k \Gamma_{\theta} / \tau$ for the flux of spectral density through a wave-number $k$, where $\tau(k)$ is the time characteristic of the cascade process at $k$ and depends upon the energy spectrum $E(k)$ (e.g. Corrsin 1961). It follows that in this range of wave-numbers (called the inertial-convective range for a scalar quantity with large Prandtl number)

$$
\frac{\partial \Gamma_{\theta}}{\partial t}+2 \lambda k^{2} \Gamma_{\theta}=-\frac{\partial}{\partial k}\left(\frac{k \Gamma_{\theta}}{\tau}\right)
$$

where the left-hand side is taken to be negligible under the usual equilibrium assumption which leads to $k \Gamma_{\theta} / \tau=$ const. A physical interpretation of the interaction between the velocity and scalar fields that produces the flux of spectral density in wave-number space is that Fourier components of $\theta$ have their scale reduced on balance by the straining field associated with the turbulent eddies of larger scale. Since the equation for $\theta$ is linear, Fourier components of $\theta$ interact with the velocity field and not directly with themselves. The assumption we now make is that as far as the flux through wave-number $k$ is concerned, it is sufficient as a reasonable approximation to measure the straining action of the turbulence by the vorticity in wave-numbers less than $k$, so that we may write

$$
\tau(k)=c\left[2 \int_{0}^{k} k^{2} E(k) d k\right]^{-\frac{1}{2}},
$$

where $c$ is a constant of order unity. In the inertial-convective range, (2.3) is equivalent to $\tau \propto k^{-\frac{3}{2}} E^{-\frac{1}{2}}$ and the well-known $\Gamma_{\theta} \propto k^{-\frac{5}{3}}$ follows from the equilibrium assumption.

Now the vorticity spectrum has a fairly sharp maximum near $k=0 \cdot 1 k_{d}$, and hence $\tau(k) \doteqdot \tau(\infty)$ for $k>k_{d}$. Hence (2.2) and (2.3) are consistent with (2.1) if $\gamma=-c^{-1}(\epsilon / \nu)^{\frac{1}{2}}$. It therefore seems to be a plausible assumption that (2.2) together with (2.3) holds for large Prandtl numbers as an approximation for all wave-numbers in the equilibrium range, and possibly for part of the energycontaining range, at least in order of magnitude. Howells (1960) has also proposed a similar equation, but an additional Heisenberg-type eddy conductivity together with a modification to make the equation valid for all Prandtl numbers were included. Equation (2.2) may be supposed to hold for the kinetic energy spectrum of the turbulence with $\Gamma_{\theta}(k)$ replaced by $E(k)$ to give a soluble equation for $E(k)$. This assumption has been termed the modified Obukhov theory by Ellison (1962) who has examined its consequences. (It is superior to the usual Obukhov theory, and also to the Heisenberg and Kovásznay theories, in that it gives an exponential decay for very large wave-numbers, but there are suspicions, described in the Appendix to $\mathrm{S}$, that the transfer of energy in the viscous subrange is not adequately described by any of these theories.) 
An equation for the spectrum function $\Gamma_{G}(k)$ of the vector quantity $\mathbf{G}=\nabla \theta$ may be derived immediately since $\Gamma_{G}=k^{2} \Gamma_{\theta}$. Thus

$$
\frac{\partial \Gamma_{G}}{\partial t}+2 \lambda k^{2} \Gamma_{G}=\frac{2 \Gamma_{G}}{\tau}-\frac{\partial}{\partial k}\left(\frac{k \Gamma_{G}}{\tau}\right)
$$

If (2.2) is a reasonable approximation throughout the equilibrium range, then so must be (2.4); and the two terms on the right-hand side are, respectively, adequate descriptions of the amplification and distortion of Fourier components of $\mathbf{G}$ by the straining motion of the turbulence.

We now turn to the magnetic field $\mathbf{H}$ and suppose in the first instance that the Lorentz forces are negligible. Then it was argued in $\mathrm{S}$ that the magnetic energy spectrum function $\Gamma(k)$, defined so that

$$
\overline{\mathbf{H}^{2}}=\int_{0}^{\infty} \Gamma(k) d k, \dagger
$$

satisfies for wave-numbers somewhat greater than $k_{d}$ the equation

$$
\frac{\partial \Gamma}{\partial t}+2 \lambda k^{2} \Gamma=2 \alpha \Gamma+\frac{\partial}{\partial k}(\gamma k \Gamma),
$$

where $\gamma$ has the same meaning as before and $\alpha(>0)$ measures the rate of extension of material lines and is proportional to $(\varepsilon / \nu)^{\frac{1}{2}}$. The parameter $\sigma=-\alpha / \gamma$ measures the relative importance of the amplification of Fourier components of $\mathbf{H}$ by the stretching of lines of force and the decrease in scale caused by the straining which eventually determines the amount of Ohmic dissipation. It is now natural to suppose by analogy with (2.4) that for all wave-numbers in the equilibrium range

$$
\frac{\partial \Gamma}{\partial t}+2 \lambda k^{2} \Gamma=\frac{2 \sigma \Gamma}{\tau}-\frac{\partial}{\partial k}\left(\frac{k \Gamma}{\tau}\right)
$$

where $\tau$ is again given by (2.3) except that the constant $c$ may have a different value, and for full generality $\sigma$ is now to be regarded as a function of wavenumber which takes the value $-\alpha / \gamma$ for $k$ somewhat greater than $k_{d}$.

It should be stressed that (2.7) is being put forward only as an approximation to the complex phenomenon of the interaction of Fourier components, in order to investigate the qualitative nature of the spectrum and to estimate the growth of magnetic energy. Insofar as (2.7) appears to contain in a consistent manner the physical processes that control the spectrum, namely amplification by stretching and decrease of scale by convection, it is believed to be a reasonable working hypothesis for the determination of the gross features of the random magnetic field. The presence of $\sigma$ in (2.7), which distinguishes this equation from (2.4) for $\Gamma_{G}$, represents the fact that $\mathbf{G}$ is amplified by the compressive, scalereducing features of the straining motion, whereas $\mathbf{H}$ is amplified by the extensive properties.

Let us now consider how (2.7) may be modified by the back reaction of the magnetic field on the turbulence. The Lorentz forces may be regarded as equiva-

+ The notation in $\mathrm{S}$ was different from that employed here, the spectrum functions of $\theta$, $\mathbf{G}$ and $\mathbf{H}$ being denoted there by $\Gamma, \Psi_{G}, \Psi_{H}$, respectively. 
lent to an isotropic pressure plus a tension along the lines of force. The pressure term may be added to the fluid pressure and is not expected to modify the straining motion that amplifies and distorts the magnetic field. The tension will oppose and restrict the stretching of the lines of force and reduce or destroy the amplification effect, but on the other hand the tension may be expected to have a much smaller effect on the properties of the straining field that distort the distribution of $\mathbf{H}$ and produce a flux of spectral density towards large wavenumbers. It is perhaps useful to think of the analogy between the lines of force and elastic strings convected by a turbulent fluid. The distortion or spectral density flux is associated with the knotting or jumbling up of the strings, and this process remains possible even if the strings are under large tension and are inextensible. Or looking at it another way, the tension in the lines of force will tend to make the local straining motion two-dimensional in a plane perpendicular to the lines of force, but the motion can still mix up the way in which $\mathbf{H}$ varies over the plane and increase the gradients of $\mathbf{H}$ in the plane while not affecting directly the strength of $\mathbf{H}$. Even when modified by magnetic forces, it does not seem that the turbulence can do anything to the magnetic field except amplify and distort. $\dagger$ It therefore seems plausible to suppose that (2.7) remains valid when magnetic forces affect the turbulence, where now $\sigma$ decreases as the magnetic energy increases and, in view of what has been said above, $\tau$ is to a first approximation directly independentof the magnetic energy and is still given by (2.3). There will, however, be an indirect dependence of $\tau$ on the magnetic energy since the magnetic forces will affect $E(k)$.

Equation (2.7) is the basis of the present work and we shall now proceed to investigate its solution, first with the magnetic forces negligible and then with these taken into account.

\section{The magnetic energy spectrum when Lorentz forces are negligible}

The quantities $\sigma$ and $\tau$ are functions of $k$ alone if the back reaction of the magnetic field is negligible and the turbulence is statistically stationary. For wave-numbers greater than $k_{d}$, we may put $\tau=-\gamma^{-1}$ and $\sigma=\sigma_{\infty}$ (constant). Then it was shown in $\mathbf{S}$ that the small-scale structure of $\mathbf{H}$ is stable in the sense that any disturbance ultimately decays to zero if $\sigma_{\infty}<\frac{5}{2}$, and argued that a steady state is then possible only if there is an input of spectral density into the large wave-numbers from smaller wave-numbers. However, it was pointed out that the large wave-number spectrum could increase with time for all $\sigma_{\infty}$ if the spectrum at smaller wave-numbers was growing, and it is now pertinent to inquire whether this is possible in the inertial subrange $L^{-1} \ll k \ll k_{d}$.

We shall assume the results of the Kolmogorov theory for the energy spectrum, from which it follows that in the inertial subrange $\tau$ and $\sigma$ are functions of $\epsilon$ and $k$. Then from dimensional considerations,

$$
\tau=\epsilon^{-\frac{1}{3}} k^{-\frac{2}{3}}, \quad \sigma=\text { const. }=\sigma_{i}, \text { say. }
$$

$\dagger$ For turbulence in a uniform strong magnetic field, a significant transfer of energy in wave-number space may be caused by non-linear interactions of Alfvén waves; but when the magnetic field is generated mainly by the turbulence itself, it seems reasonable to assume that non-local transfer in wave-number space due to such interactions is negligible. 
Moreover, the magnetic diffusivity may be neglected, and it is easy to show that the general unsteady solution of $(2.7)$ is

$$
\Gamma(k, t)=\left(k_{0} / k\right)^{\frac{5}{3}-2 \sigma_{i}} \Gamma(k, 0), \quad \text { where } \quad k_{0}=\left[{ }_{3}^{2} \epsilon^{\frac{1}{3}} t+k^{-\frac{3}{2}}\right]^{-\frac{2}{3}} .
$$

Thus the asymptotic behaviour of $\Gamma(k, t)$ at a fixed value of $k$ depends on the initial value near $k=0$, so that amplification or decay in the inertial subrange is not determined by the local (in wave-number) conditions but is again decided by the input from smaller wave-numbers. However, if we suppose that $\Gamma \sim k^{4}$ for small $k$, as is implied by kinematical requirements, it follows from (3.2) that the spectrum function ultimately decays if

$$
\sigma_{i}<\frac{17}{6}
$$

If this condition is not satisfied, the inertial subrange eddies would be unstable to the introduction of a small magnetic field and the magnetic energy would grow until checked by the back reaction. (A turbulent dynamo would then be possible, controlled by the structure of the inertial subrange eddies.)

However, the value of $\sigma_{i}$ may be inferred from the analogy between $\mathbf{H}$ and the random vorticity $\boldsymbol{\omega}$, based on the observation by Batchelor that $\mathbf{H}$ and $\boldsymbol{\omega}$ satisfy the same equation if the diffusive terms are neglected, as is appropriate for the inertial subrange when $\lambda \leqslant v$. For according to the modified Obukhov theory, the spectrum function of vorticity satisfies (2.7) with $\sigma=1$ (and $\lambda=\nu$ ), which implies that

$$
\sigma_{i}=1
$$

The analogy with vorticity is further supported by the exact solution $\mathbf{H} \propto \omega$ that exists when $\lambda=\nu$, as first observed by Batchelor. If $\sigma_{i} \neq 1$ and yet $\sigma_{i}$ is independent of $\lambda$, it would have to be concluded, as appears unlikely, that this solution is a very special one and that the general solution for $\mathbf{H}$ when $\lambda=v$, has different statistical properties. $\dagger$ A further reason for (3.4) is given below. Nevertheless, the analogy between $\mathbf{H}$ and $\boldsymbol{\omega}$ is not absolutely convincing because of the special relation $\boldsymbol{\omega}=\operatorname{curl} \mathbf{u}$ and the fact that the vector potential $\mathbf{A}$ (where $\mathbf{H}=\operatorname{curl} \mathbf{A}$ ) does not satisfy the same equation as $\mathbf{u}$ (see Moffatt 1961), and it cannot be altogether ruled out that (3.4) may not be correct. But be this as it may, we shall assume throughout this paper that (3.4) is satisfied (at least in the absence of significant back reaction).

It follows that (3.3) is satisfied, and if it is also assumed that $\sigma_{\infty}<\frac{5}{2}$ (reasons were in fact given in $\mathrm{S}$ for believing that $\sigma_{\infty}<1$ ), then the equilibrium range of wave-numbers is magnetically stable and cannot maintain indefinitely a magnetic field without an input of magnetic energy. It is now a simple matter to determine the spectrum in the equilibrium range for a statistically steady state maintained by an input of magnetic energy on the scale $L$. The steady solution of (2.7) is

$$
\Gamma(k)=\tau P(k) / k,
$$

+ It is perhaps worth mentioning here that the special exact solution with $\lambda=\nu$ cannot be taken to imply that a magnetic field can maintain itself against Ohmic dissipation without an input of magnetic energy, for it is necessary to supply vorticity to maintain a stationary turbulent velocity field. If the mechanical body forces $\mathbf{F}$ that drive the turbulence are irrotational, and therefore make no contribution to the vorticity equation, they would be equivalent to a random hydrostatic pressure and do no net work. 
where $P(k)$ satisfies the equation

$$
\frac{\mathrm{I}}{P} \frac{d P}{d k}=\frac{2 \sigma}{k}-2 \lambda k \tau
$$

Thus in the inertial subrange,

$$
\Gamma(k)=\chi_{i} \epsilon^{-\frac{1}{3}} k^{\frac{1}{3}}, \dagger
$$

and for wave-numbers somewhat greater than $k_{d}$,

$$
\Gamma(k)=\chi_{\infty}(-\gamma)^{-1} k^{2 \sigma_{\infty}-1} e^{\lambda k^{2} / \gamma},
$$

where $\chi_{i}$ and $\chi_{\infty}$ depend upon the amount of magnetic energy fed into the spectrum at small wave-numbers (and are not necessarily of the same dimensions).

It may be supposed that (3.6) and (3.7) agree in order of magnitude at $k=k_{l d}$. Hence

$$
\chi_{\infty} \approx-\gamma \epsilon^{-\frac{1}{3}} k_{d}^{-2 \sigma_{\infty}+\frac{4}{3}} \chi_{i}
$$

As a further rough approximation, we may use Moffatt's method to estimate $\chi_{i}$ in terms of the large-scale magnetic field $\mathbf{H}_{0}$ that would be present in the absence of turbulence. We take the mean square value of this field as

$$
\overline{H_{0}^{2}} \doteqdot \int_{0}^{L^{-1}} \Gamma(k) d k \doteqdot L^{-1} \Gamma\left(L^{-1}\right)
$$

and suppose further that (3.7) holds down to $L^{-1}$ at least in order of magnitude. Then

$$
\chi_{i}=H_{0}^{2} e^{\frac{1}{3}} L^{\frac{4}{3}} \text {. }
$$

The spectrum is sketched in figure 1 on a logarithmic scale. There is an exponential cut-off for $k>k_{c}$, the conduction wave-number $\left(\epsilon / \nu \lambda^{2}\right)^{\frac{1}{4}}$.

The spectrum in the viscous-convective range $k_{d} \ll k \ll k_{c}$ is determined by the straining motion of the small eddies that make the major contribution to the vorticity and is independent of the magnetic diffusivity. If $\sigma_{\infty}>\frac{1}{2}$, the spectrum has the expected shape reaching a maximum at the conduction wave-number. But if $\sigma_{\infty}<\frac{1}{2}$, the maximum occurs near $k=k_{d}$, as for the vorticity, but the tail of the magnetic spectrum extends very much further and indeed contains most of the magnetic energy.

To obtain an estimate of the mean-square magnetic field, we break up the range of integration for (2.5) into the three intervals $\left(0, L^{-1}\right),\left(L^{-1}, k_{d}\right),\left(k_{d}, \infty\right)$, and substitute (3.7) and (3.8) into the second and third ranges, respectively. Thus,

$$
\overline{\mathbf{H}}^{2} \doteqdot \overline{H_{0}^{2}}+{ }_{4}^{3} \chi_{i} \epsilon^{-\frac{1}{3}}\left(k_{d}^{\frac{4}{3}}-L^{-\frac{4}{3}}\right)+\frac{\chi_{\infty}}{2 \gamma \sigma_{\infty}} k_{d}^{2 \sigma_{\infty}}-\frac{\chi_{\infty}\left(\sigma_{\infty}-1\right) !}{2 \gamma}\left(-\frac{\gamma}{\lambda}\right)^{\sigma_{\infty}}
$$

For $\lambda$ small and $\sigma_{\infty}$ not too close to zero, the last term dominates and it follows from (3.9) and (3.11) that

$$
\overline{\mathbf{H}^{2}}=\frac{1}{2} c^{-\sigma_{\infty}}\left(\sigma_{\infty}-1\right) !(\nu / \lambda)^{\sigma_{\infty}} R \overline{H_{0}^{2}},
$$

where $c=\left(\nu / \epsilon \gamma^{2}\right)^{\frac{1}{2}}$ and $\left(k_{d} L\right)^{\frac{4}{3}}=R$, the Reynolds number of the turbulence based on the energy-containing eddies.

$\dagger$ For general $\sigma_{i}$, the exponent of $k$ is $2 \sigma_{i}-\frac{5}{3}$. Moffatt (1961) has given an argument based on the vector potential $A$ to show that the exponent should be $\frac{1}{3}$, which is a further reason for taking $\sigma_{i}=1$. However, Moffatt's argument assumes that ' $\mathbf{A}^{2}$-stuff' is generated on the length-scale $L$ and is then transferred through the spectrum until dissipated at large wave-numbers, like kinetic energy, and it is not absolutely certain that ' $\mathbf{A}^{2}$-stuff' is not created in the inertial subrange, in which case the argument fails. 
The rate at which extra energy must be fed into the system to balance the loss due to Ohmic dissipation is

$$
D=\lambda \mu \int_{0}^{\infty} k^{2} \Gamma(k) d k
$$

per unit volume ( $\mu=$ permeability, M.K.s. units are employed). This integral is dominated by the contribution from wave-numbers greater than $k_{d}$, and it follows that

$$
D=\mu \chi_{\infty}\left(\frac{\nu}{\lambda}\right)^{\sigma_{\infty}}=\left(\frac{\epsilon}{\nu}\right)^{\frac{1}{2}} \mu \overline{\mathbf{H}^{2}}
$$

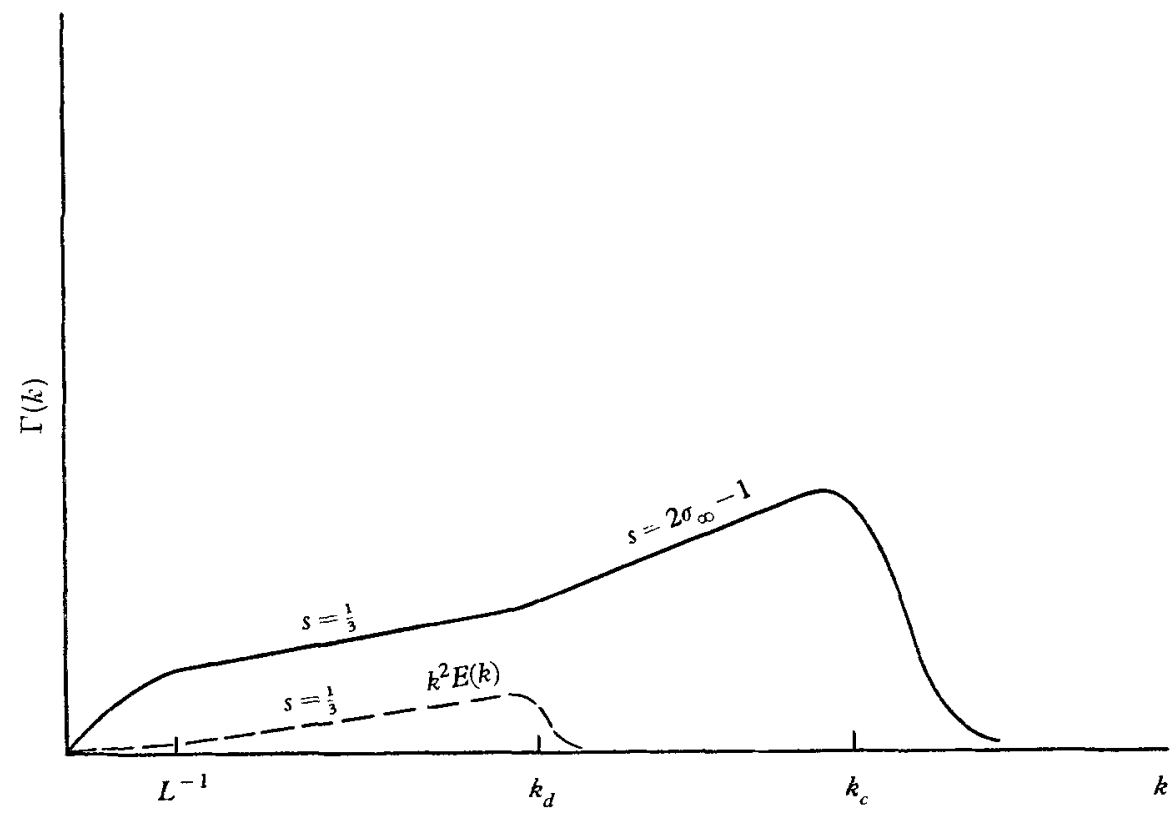

FIGURE 1. Sketch on a logarithmic scale of the magnetic spectrum function with Lorentz forces negligible. The letter $s$ denotes the slope. $k_{d}=\left(\epsilon / \nu^{3}\right)^{\frac{1}{2}} . k_{c}=\left(\epsilon / \nu \lambda^{2}\right)^{\frac{1}{4}}$. The dashed curve is the vorticity spectrum (not on the same scale).

provided $\sigma_{\infty}$ is not too small, factors of order unity being neglected. The rate of degradation of magnetic energy on the length-scale $L$ by interaction with the energy containing eddies is of order

$$
\mu \overline{H_{0}^{2}} u^{\prime} / L=D_{0}, \text { say, }
$$

where $u^{\prime}$ is a root-mean-square turbulent velocity component. This is the rate at which magnetic energy must be supplied to maintain a steady magnetic spectrum.

$$
\frac{D_{0}}{D}=\left(\frac{\lambda}{\nu}\right)^{\sigma_{\infty}} R^{-\frac{3}{2}} \ll 1
$$

thus the energy lost through Ohmic dissipation comes from the turbulence. The assumption that the magnetic forces do not modify significantly the turbulence is consistent provided $D \ll \rho \epsilon$, where $\rho$ is the density of the fluid. This condition is equivalent to the magnetic energy density $\frac{1}{2} \mu \overline{\mathbf{H}^{2}}$ being small compared 
with $\rho(\epsilon v)^{\frac{1}{2}}$, the kinetic energy density of the small eddies, which in turn implies from (3.13) that

$$
\mu \widehat{H_{0}^{2}} \ll \rho u^{\prime 2} R^{-\frac{3}{2}}(\lambda / v)^{\sigma_{\infty}} .
$$

If the applied field does not satisfy this requirement, the neglect of the Lorentz forces is not justified.

\section{The action of Lorentz forces}

Let us suppose now that the condition (3.17) is not satisfied, so that the interaction between the magnetic field and the turbulence becomes important before Ohmic dissipation can limit the growth by itself. In the first instance, we shall assume and later check for consistency that in the equilibrium state the magnetic energy density does not greatly exceed the kinetic energy density of the small, energy-dissipating eddies, so that the inertial subrange eddies are unaffected by the magnetic forces. In view of the earlier discussion in $\$ 2$, the magnetic forces then decrease $\sigma$ for the small eddies and leave the relation between $\tau$ and $E(k)$ unaltered, at least to a sufficient approximation.

Since there is now a back reaction on the turbulence, care is needed in the definition of $\epsilon$. We shall define $\epsilon$ to be the rate at which energy is dissipated by viscosity per unit mass, so that $\overline{\omega^{2}}=\epsilon / \nu$, and the values of $\gamma$ and $c$ are unaffected according to our assumptions. Also, the definition $k_{d}=\left(\epsilon / \nu^{3}\right)^{\frac{1}{4}}$ is retained, and it will be assumed that the vorticity spectrum has a peak near $k=k_{d}$. The rate of transfer of kinetic energy density through wave-numbers in the inertial subrange is denoted by $\epsilon_{i}$, and is of order $u^{\prime 3} / L$. In general, $\epsilon_{i} \neq \epsilon$, but in fact we shall see that the difference is not significant for the case under present consideration, and is indeed negligible if $\log (v / \lambda)$ is large.

For wave-numbers somewhat greater than $k_{d l}$, the action of the Lorentz forces may be represented by a dependence of $\sigma_{\infty}$ upon $\mu \overline{\mathbf{H}^{2}} / \rho(\epsilon \nu)^{\frac{1}{2}}=Z$, say, of the form

$$
\sigma_{\infty}=F(Z)
$$

where $F^{\prime}(Z)$ decreases to zero as $Z$ increases. According to the present model, an input of magnetic energy is still necessary to maintain a steady state, if necessary when Lorentz forces are negligible. For given $\overline{H_{0}^{2}}$ on the scale $L$, the magnetic energy in the equilibrium state is determined in principle as follows. The magnetic spectrum is still given by (3.7) and (3.8) together with (3.9) and (3.11), provided $\epsilon$ is replaced by $\epsilon_{i}$ wherever it appears explicitly in these expressions. The magnetic energy density is then given roughly by (3.12) (with $\epsilon_{i}$ instead of $\epsilon$ in the second term), and the Ohmic dissipation rate $D$ may likewise be evaluated. Then the conservation of energy requires that $D=\rho\left(\epsilon_{i}-\epsilon\right)$, and the elimination of $\sigma_{\infty}$ by (4.1) gives a complex, non-linear equation for $\overline{\mathbf{H}}^{\overline{2}}$ in terms of $\overline{H_{0}^{2}}$.

A simplification is possible for the case when $Z$ is of order unity if it is assumed that the stretching due to the small eddies is then almost completely eliminated so that $\sigma_{\infty}$ is very small. Equation (3.12) may be rewritten

$$
\overline{\mathbf{H}^{2}} \doteqdot \frac{3}{4} R \overline{H_{0}^{2}}+\frac{1}{2} R \overline{H_{0}^{2}}\left[\left(\sigma_{\infty}-1\right) !(\nu / c \lambda)^{\sigma_{\infty}-1}\right]
$$


where the first term comes from the range $\left(L^{-1}, k_{d}\right)$ and the second from $\left(k_{d}, \infty\right)$. When $\sigma_{\infty}$ is small compared with $\{\log (\nu / \lambda)\}^{-1}$, the second term is approximately

and

$$
\begin{gathered}
\frac{1}{2} R \overline{H_{0}^{2}} \log (\nu / \lambda), \\
\overline{\mathbf{H}^{2}} \doteqdot\left(\frac{1}{2} \log (\nu / \lambda)+\frac{3}{4}\right) R \bar{H}_{0}^{2},
\end{gathered}
$$

which is now independent of $\sigma_{\infty}$. This then is the amplification if the magnetic forces resist sufficiently the stretching by the small eddies $\dagger$ but do not affect the inertial subrange components. The magnetic spectrum for $k_{d} \ll k \ll k_{c}$ now has a $k^{-1}$ behaviour, and the fairly flat maximum of the spectrum occurs near $k=k_{d}$, having a $k^{\frac{1}{3}}$ dependence for $k \ll k_{d}$ (see figure 2 ).

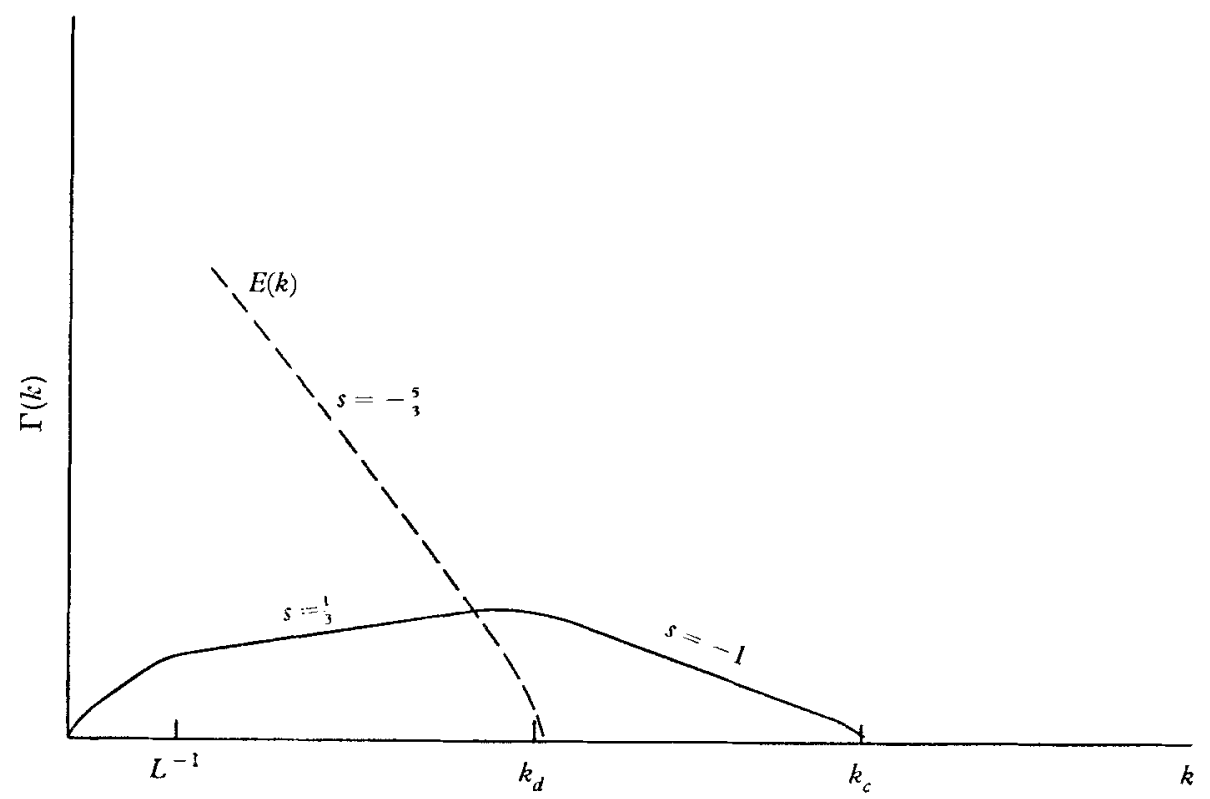

FIGURE 2. Sketch on a logarithmic scale of the magnetic spectrum function when Lorentz forces suppress the amplification by the small eddies. The dashed curve is the energy spectrum (not on the same seale).

The presence of $\lambda$ in (4.4) is at first sight odd, but it arises because a steady state has been assumed. According to the present model, spectral density is being continually transferred through wave-number space until it is destroyed near $k=k_{c}$, and the amount of magnetic energy in components with $k>k_{d}$ depends upon the value of $\lambda$.

For (4.4) to be consistent, it is necessary that $\mu \overline{\mathbf{H}^{2}} \approx \rho(\epsilon v)^{\frac{1}{2}} \approx \rho u^{\prime 2} R^{-\frac{1}{2}}$, i.e.

$$
\mu \overline{H_{0}^{2}} \approx \rho u^{\prime 2} R^{-\frac{3}{2}}\left[\log (\nu / \lambda)+\frac{3}{2}\right]^{-1} .
$$

Thus if

$$
\rho u^{\prime 2} R^{-\frac{3}{2}}\left(\frac{\lambda}{\nu}\right)^{\sigma_{\infty}^{(0)}}<\mu \overline{H_{0}^{2}} \text { 雨 } \rho u^{\prime 2} R^{-\frac{3}{2}}\left[\log \left(\frac{\nu}{\lambda}\right)+\frac{3}{2}\right]^{-1},
$$

$\dagger$ It can be deduced from equation (3.26) of $S$ that a small magnetic disturbance of length-scale $<k_{d}^{-1}$ is not amplified if $\sigma_{\infty}<\lambda / \nu$, and hence the condition on $\sigma_{\infty}$ for (4.3) to hold is reasonable. 
where $\sigma_{\infty}^{(0)}$ denotes $F(0)$, the amplification of the magnetic field is modified by the back reaction on the small eddies and lies between the values (3.13) and (4.4).

When $\sigma_{\infty}$ is close to zero, the Ohmic dissipation per unit volume is

$$
D=\mu \chi_{\infty}=-\gamma \mu \overline{\mathbf{H}^{2}}\left[\frac{1}{2} \log \left(\frac{\nu}{\lambda}\right)+\frac{3}{4}\right]^{-1} \approx \rho \epsilon\left[\frac{1}{2} \log \left(\frac{\nu}{\lambda}\right)+\frac{3}{4}\right]^{-1},
$$

if $\mu \overline{\mathbf{H}^{2}} \approx \rho(\epsilon \nu)^{\frac{1}{2}}$. Thus $\epsilon_{i} \doteqdot \epsilon$ if $\log (\nu / \lambda)$ is appreciable, as stated earlier.

\section{The further action of the Lorentz forces}

If $\overline{H_{0}^{2}}$ is significantly greater than the value given by (4.5), the amplification allowed by (4.4) gives a magnetic energy density appreciably greater than that of the small eddies, and it must be concluded that the Lorentz forces are modifying the inertial subrange eddies. The situation is now even more complicated, and in order to extract the main features of the phenomenon, fairly drastic simplifications will be made.

We introduce a wave-number $k_{f}$, that is assumed to lie in the inertial subrange, with the property that the Fourier components of the turbulence with wavenumber greater than $k_{f}$ have the same energy as the magnetic field, i.e.

$$
\frac{1}{2} \mu \overline{\mathbf{H}}^{2}=\int_{k_{\rho}}^{\infty} E(k) d k .
$$

It is now supposed that the tension in the lines of force destroys the amplification due to stretching for components of the magnetic field with wave-numbers somewhat greater than $k_{f}$, i.e. $\sigma=0$ for $k \gg k_{f}$, but that the value of $\sigma$ for $L^{-1} \ll k \ll k_{f}$ is unaltered and has the value $\sigma_{i}=1$.

It is convenient to break up the equilibrium range into three subranges: subrange A specified by $L^{-1} \ll k \ll k_{f}$; subrange $\mathrm{B}, k_{f} \ll k \ll k_{d}$; subrange $C$, $k_{i} \ll k$. Diffusive effects are negligible in subranges $\mathrm{A}$ and $\mathrm{B}$, and the energy spectrum is damped out by viscosity in subrange C. Our equation (2.7) for $\Gamma(k)$ involves the variable time-scale $\tau(k)$, which in turn depends upon $E(k)$. Since total energy is conserved, it is plausible to suppose here that in the inertial subrange $E(k)$ satisfies the equation

$$
\frac{\partial E}{\partial t}=-\frac{\partial S}{\partial k}-\frac{\mu \sigma \Gamma}{2 \tau}
$$

where $S(k)$ is the flux of kinetic energy through wave-number $k$. The second term on the right-hand side of (5.2) describes the energy extracted from the turbulence by the magnetic field. When a steady state is set up, the left-hand side of (5.2) is zero.

Now it is assumed that the magnetic forces are negligible in subrange $A$ and $\sigma=0$ in subrange $\mathrm{B}$. Hence $S$ is constant in subranges $\mathrm{A}$ and $\mathrm{B}$. There is no net transfer of energy between components of magnetic field and kinetic energy for $k \gg k_{f}$, and hence the value of $S$ in subrange $\mathrm{B}$ is the rate at which kinetic energy is dissipated by viscosity per unit mass. In accordance with the notation of the previous section, we denote this by $\epsilon$. The value of $S$ in subrange $A$ is denoted 
by $\epsilon_{i}$. As before, $\epsilon_{i}$ represents the loss of energy by the large-scale components of the turbulence and is of order $u^{\prime 3} / L$. The difference between $\epsilon_{i}$ and $\epsilon$ is the rate at which energy is extracted from the turbulence by the magnetic field. Clearly,

$$
\epsilon_{i}-\epsilon=\int_{A}^{B} \frac{\mu \sigma \Gamma}{2 \tau} d k
$$

demonstrating that in the present model the net transfer of energy between components of $\mathbf{u}$ and $\mathbf{H}$ occurs mainly in the vicinity of wave-number $k_{f}$.

If we now assume that, in subranges $\mathrm{A}$ and $\mathrm{B}, E(k)$ depends only on $S$ and $k$, it follows that

$$
\left.\begin{array}{l}
\text { in } \mathrm{A}, \quad E(k)=K_{i} \epsilon_{i}^{\frac{2}{3}} k^{-\frac{5}{3}}, \\
\text { in } \mathrm{B}, \quad E(k)=K \epsilon^{\frac{2}{3}} k^{-\frac{5}{3}},
\end{array}\right\}
$$

where $K_{i}$ and $K$ are numerical constants. Thus the shape of the energy spectrum is unaltered. The vorticity spectrum then retains a maximum near $k=k_{d}$ and the value of $\tau$ in the three subranges may be approximated by

$$
\left.\begin{array}{l}
\text { in } \mathrm{A}, \quad \tau=\epsilon_{i}^{-\frac{1}{3}} k^{-\frac{2}{3}}, \\
\text { in } \mathrm{B}, \quad \tau=\epsilon^{-\frac{1}{3}} k^{-\frac{2}{3}}, \\
\text { in } \mathrm{C}, \quad \tau=-\gamma^{-1} \approx(\nu / \epsilon)^{\frac{1}{2}},
\end{array}\right\}
$$

provided $\epsilon$ is not an order of magnitude less than $\epsilon_{i}$, as will be verified later. It follows from (2.7), with the assumption of a steady state maintained by a field $\mathbf{H}_{0}$ on scale $L$ and the insertion of the appropriate values of $\sigma$, that

$$
\begin{aligned}
& \text { in } \mathrm{A}, \quad \Gamma(k)=\chi_{i} \epsilon_{i}^{-\frac{1}{3}} k^{\frac{1}{3}}, \\
& \text { in } \mathrm{B}, \quad \Gamma(k)=\chi_{B} e^{-\frac{1}{3}} k^{-\frac{5}{3}}, \\
& \text { in } \mathrm{C}, \quad \Gamma(k)=-\gamma^{-1} \mathcal{X}_{\infty} k^{-1} e^{\lambda k^{2} / \gamma} .
\end{aligned}
$$

Since there is no net amplification of magnetic energy in components with $k \gg k_{f}, \frac{1}{2} \mu \chi_{B}$ is the flux of magnetic energy through wave-numbers in the range $\mathrm{B}$ and must be equal to $\frac{1}{2} \mu \chi_{\infty}$ and must also be equal to the Ohmic dissipation $D$. Substituting (5.6c) into the integral (3.14) for $D$, as is appropriate since the dissipation is dominated by the contribution from subrange $\mathrm{C}$, we find that $\chi_{B}=\chi_{\infty}$ is consistent and, moreover, we observe that $(5.6 b)$ and $(5.6 c)$ join smoothly at $k=k_{d}$, each being of order $\chi_{\infty} \nu^{\frac{5}{4}} \epsilon^{-\frac{3}{4}}$. Also, it is still permissible, as a rough approximation, to estimate $\chi_{i}$ by (3.11) (with $\epsilon_{i}$ replacing $\epsilon$ ), and to suppose that (5.6a) and $(5.6 b)$ join smoothly, so that

$$
\chi_{i} \epsilon_{i}^{-\frac{1}{3}} \approx \chi_{B} \epsilon^{-\frac{1}{3}} k_{f}^{-2} \text {. }
$$

The kinetic energy in components with $k>k_{f}$ is of order $\rho \epsilon^{\frac{2}{3}} k_{f}^{-\frac{2}{3}}$. The meansquare magnetic field may be calculated roughly as for (3.12) and is

$$
\overline{\mathbf{H}^{2}} \doteqdot \frac{3}{4} \chi_{i} \epsilon_{i}^{-\frac{1}{3}} k_{f}^{\frac{4}{3}}+\frac{3}{2} \chi_{B} \epsilon^{-\frac{1}{3}} k_{f}^{-\frac{2}{3}}-\frac{\chi_{\infty}}{2 \gamma} \log \left(\frac{\nu}{\lambda}\right)
$$

where the terms in this expression are the contributions from subranges $A, B$ and $\mathrm{C}$, respectively. Using (5.7), we may rewrite (5.8) as

$$
\overline{\mathbf{H}^{2}} \approx \frac{5}{2} \chi_{\infty} \epsilon^{-\frac{1}{3}} k_{f}^{-\frac{2}{3}}\left[1+\frac{2}{5}\left(\frac{k_{f}}{k_{d}}\right)^{\frac{2}{3}} \log \left(\frac{\nu}{\lambda}\right)\right],
$$


where for definiteness we have put $\gamma=-\frac{1}{2}(\epsilon / \nu)^{\frac{1}{2}}$. Then from (5.1),

$$
\frac{5}{4} \mu \chi_{\infty}=\rho \epsilon\left[1+\frac{2}{5}\left(\frac{k_{f}}{k_{d}}\right)^{\frac{2}{3}} \log \left(\frac{\nu}{\lambda}\right)\right]^{-1} .
$$

Moreover, from the conservation of energy

$$
\epsilon_{i}=\epsilon+(\mu / 2 \rho) \chi_{\infty}
$$

since the amount of magnetic energy entering the low wave-number end of subrange A is negligible. From (5.10), it is clear that the Ohmic dissipation is at most comparable with the viscous dissipation, and hence from (5.11) it follows that $\epsilon$ is always an appreciable fraction of $\epsilon_{i}$. The statement made earlier that $\epsilon$ is not an order of magnitude less than $\epsilon_{i}$ is therefore verified.

It remains to determine $k_{f}$. From (5.7) and (3.11),

$$
\overline{H_{0}^{2}} L^{\frac{4}{3}} \approx \chi_{B} \epsilon^{-\frac{1}{3} k_{f}^{-2}} \text {. }
$$

The elimination of $\chi_{B}$ between (5.10) and (5.12) then gives an equation for $k_{f}$. In order to simplify, we shall suppose first that there is negligible energy in subrange $\mathrm{C}$ so that

$$
\left(\frac{k_{f}}{k_{d}}\right)^{\frac{2}{3}} \log \left(\frac{\nu}{\lambda}\right) \ll 1
$$

This would seem to be the case for turbulence of large Reynolds number, since $k_{f}$ is assumed to lie inside the inertial subrange, unless $\lambda$ is extremely small. Then the square brackets in (5.9) and (5.10) are approximately equal to unity. In this case, there is therefore approximate equality of Ohmic and viscous dissipation, and the ratio of mean-square vorticity and current is approximately equal to the resistivity divided by the viscosity. Neglecting factors of order unity, we find from (5.10) and (5.12) that

$$
\left(k_{f} L\right)^{2}=\rho u^{\prime 2} / \mu \bar{H}_{0}^{2},
$$

and (5.9) then gives

$$
\overline{\mathbf{H}^{2}}=\overline{H_{0}^{2}}\left(\rho u^{\prime 2} / \mu \overline{H_{0}^{2}}\right)^{\frac{2}{3}} \text {. }
$$

Since $k_{d} L=R^{\frac{3}{4}},(5.14)$ and (5.15) will be valid provided

i.e.

$$
\begin{gathered}
1 \ll k_{f} L \ll R^{3}[\log (\nu / \lambda)]^{-\frac{3}{2}}, \\
\rho u^{\prime 2} R^{-\frac{3}{2}}[\log (\nu / \lambda)]^{3} \ll \mu \overline{H_{0}^{2}} \ll \rho u^{\prime 2} .
\end{gathered}
$$

The requirement $\rho\left(\epsilon \nu^{\prime}\right)^{\frac{1}{2}} \ll \mu \overline{\mathbf{H}^{2}} \ll \rho u^{\prime 2}$ is automatically satisfied. The spectra of $\mathbf{H}$ and $\mathbf{u}$ are shown in figure 3 .

If the converse of (5.13) holds, the Ohmic dissipation is negligible, $\epsilon_{i} \doteqdot \epsilon$, $\mu \chi_{\infty} \ll \rho \epsilon$, and the magnetic energy is mostly in subrange C. A little algebra then gives

and

$$
\begin{aligned}
& \mu \overline{H_{0}^{2}}\left(\frac{k_{f}}{k_{d}}\right)^{\frac{8}{3}} \approx \rho u^{\prime 2} R^{-\frac{3}{2}}\left[\log \left(\frac{\nu}{\lambda}\right)\right]^{-1}, \\
& \overline{\mathbf{H}^{2}} \approx \overline{H_{0}^{2}}\left(\frac{\rho u^{\prime 2}}{\mu \overline{H_{0}^{2}}}\right)^{\frac{3}{4}} R^{-\frac{1}{8}}\left[\log \left(\frac{v}{\lambda}\right)\right]^{\frac{1}{4}},
\end{aligned}
$$

valid if

$$
\rho u^{\prime 2} R^{-\frac{3}{2}}\left[\log \left(\frac{\nu}{\lambda}\right)\right]^{-1} \ll \mu \overline{H_{0}^{2}} \ll \rho u^{\prime 2} \min \left\{R^{\frac{1}{2}}\left[\log \left(\frac{\nu}{\lambda}\right)\right]^{-1}, R^{-\frac{3}{2}}\left[\log \left(\frac{\nu}{\lambda}\right)\right]^{3}\right\}
$$


by virtue of the requirement $L^{-1} \ll k_{f} \ll k_{d}$. If $R>[\log (\nu / \lambda)]^{2}$, the range $(5.19)$ lies between the ranges $(4.6)$ and (5.16).

Independently of whether (5.13) is satisfied, the magnetic spectrum in subrange $\mathrm{A}$ is smaller than the kinetic energy spectrum by the factor $\left(k / k_{f}\right)^{2}$ so that the neglect of magnetic forces on the energy spectrum in subrange $A$ is a consistent approximation.

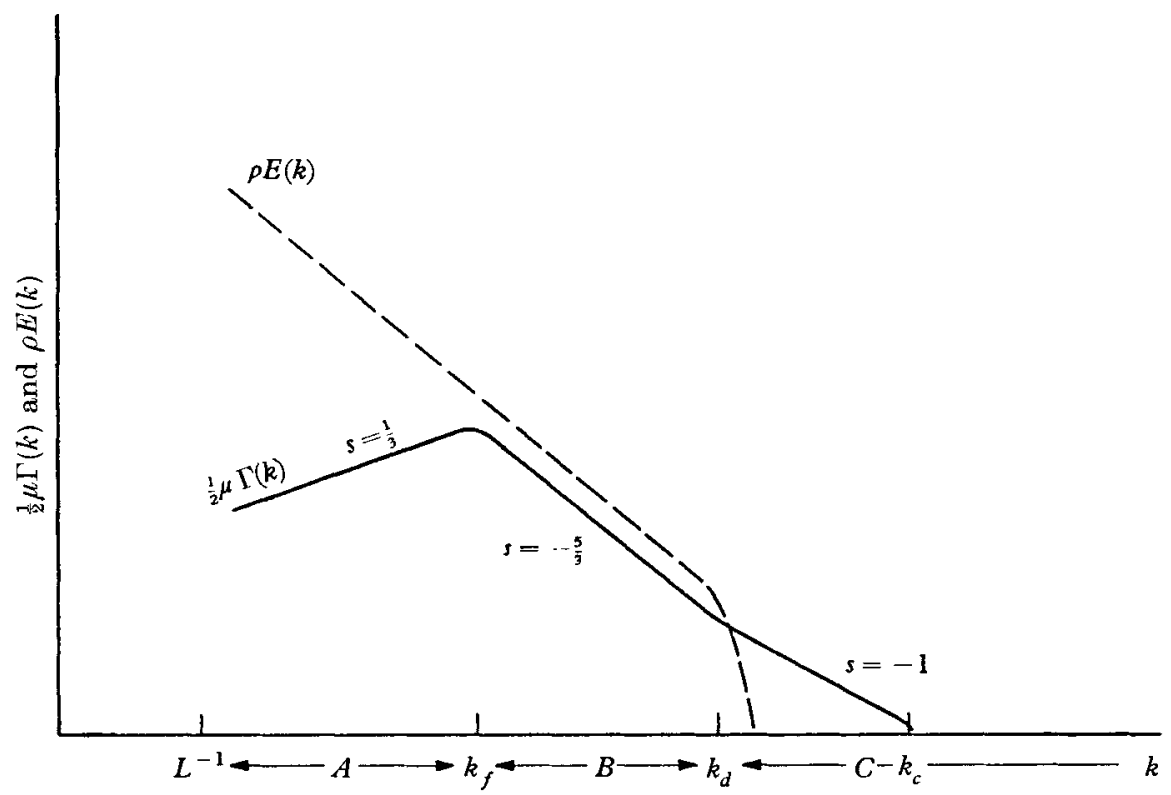

Figure 3. Sketch of the magnetic and kinetic energy spectrum functions in the equilibrium range of wave-numbers for the case when Lorentz forces affect eddies in the inertial subrange. The kinetic energy in components with $k>k_{f}$ is roughly equal to the total magnetic energy.

\section{Discussion}

In figure 4 , the dependence of $R^{-1} \overline{\mathbf{H}^{2}} / \overline{H_{0}^{2}}=Y$, say, on $R^{3} \mu \overline{H_{0}^{2}} / \rho u^{\prime 2}=X$, say, is shown schematically on a logarithmic scale according to the results $(3.13),(4.4)$, (5.15) and (5.18). The plot is Reynolds number independent for constant magnetic Prandtl number, provided of course that the Reynolds number is large enough for the inertial subrange to exist, and it is also supposed that

$$
R \gg[\log (\nu / \lambda)]^{2} .
$$

These results are based on the assumption that a statistically steady distribution of $\mathbf{H}$ is set up in the equilibrium range of wave-numbers and maintained by a permanent source of magnetic energy on a length-scale $L$. However, it would be sufficient if the time $T$ characteristic of the large-scale components of $\mathbf{H}$ was large compared with the time required for the small-scale components to reach statistical equilibrium, this latter time being of order $-\gamma^{-1} \log (\nu / \lambda)$ (see Batchelor 1959 or S). In the absence of permanent external electromotive forces, large-scale components of $H$ would be expected to persist for time $L / u^{\prime} \approx-\gamma^{-1} R^{\frac{1}{2}}$, so that in this case the assumption of a steady state in the equilibrium range 
would be valid if $R^{\frac{1}{2}} \gg \log (\nu / \lambda)$. If this last condition were violated, a study of the unsteady problem would be called for, but this is another matter.

The assumption that a quasi-steady distribution of magnetic field with wavenumbers in the equilibrium range cannot be maintained without a supply of magnetic energy from external sources is based on the hypotheses that $\sigma_{\infty}$ in the absence of a back reaction is less than $\frac{5}{2}$ (see $S$ ) and that the Lorentz forces act to decrease $\sigma$. However, the possibility that $\sigma_{\infty}>\frac{5}{2}$ cannot be altogether ruled out, in which case the magnetic energy in components with $k>k_{d}$ would increase without limit in the absence of a back reaction, and for the sake of completeness this case will now be examined.

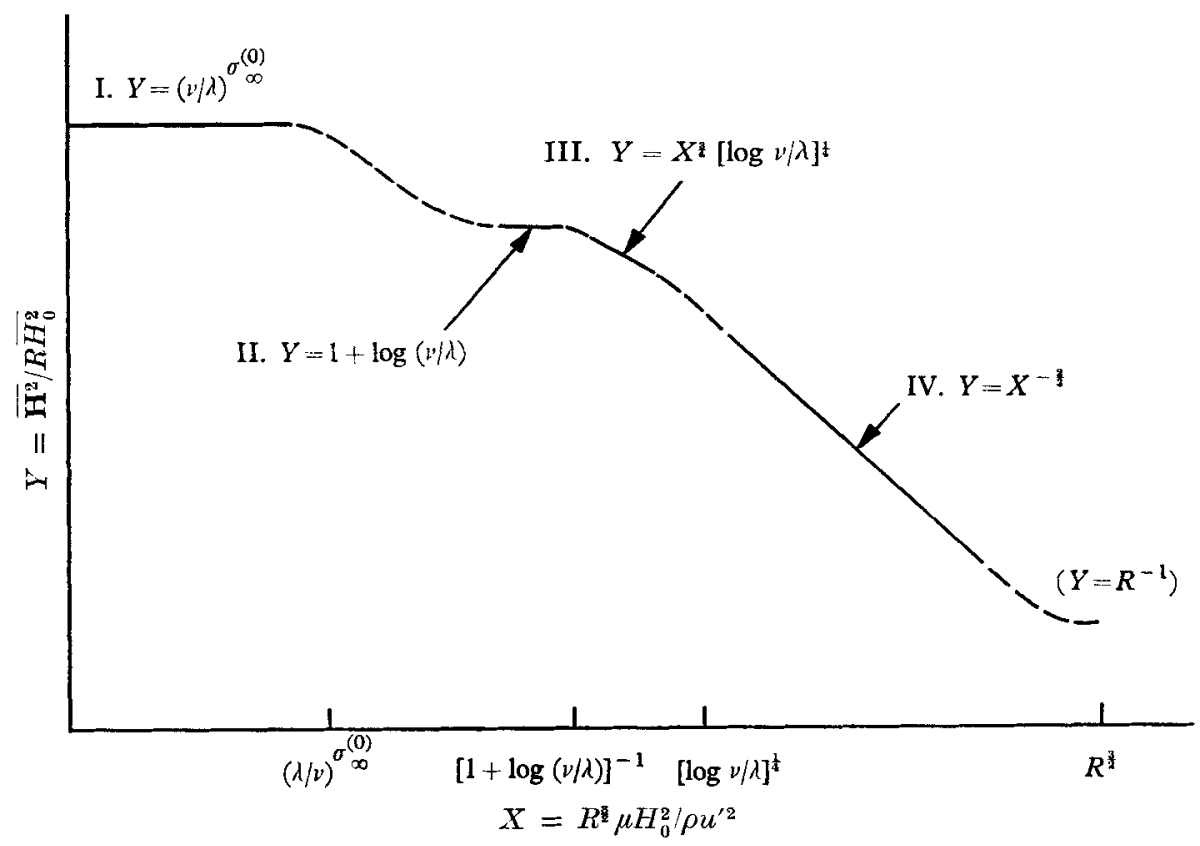

FigURE 4. Sketch on a logarithmic scale of amplification factor against energy of applied field scaled to be Reynolds number independent (factors of order unity are omitted). I, equations (3.13) and (3.17), Lorentz forces negligible; II, (4.4) and (4.5), magnetic energy comparable with kinetic energy of small eddies; III, (5.18) and (5.19), and IV, (5.15) and (5.16), inertial subrange eddies modified by Lorentz forces. Dashed curve is nterpolated. It is supposed that $R \gg[\log (\nu / \lambda)]^{2}$.

It is again assumed that the value of $\sigma_{\infty}$ is related to the ratio of the magnetic energy and kinetic energy of the small eddies by the relation (4.1). The magnetic energy of a small magnetic disturbance will continue to grow until $\sigma_{\infty}$ is reduced to the value $\frac{5}{2}$, when Ohmic dissipation balances the increase of magnetic energy by the stretching due to the small eddies. A steady state is then set up which does not require an external supply of magnetic energy (although mechanical energy is required to maintain the turbulence). This equilibrium is stable, for if $\mathbf{H}^{2}$ increases past the equilibrium value, $\sigma_{\infty}$ drops below $\frac{5}{2}$ and Ohmic dissipation exceeds the generation, so that $\overline{\mathbf{H}^{2}}$ decreases and $\sigma_{\infty}$ rises; and vice versa. Since $\sigma_{\infty}$ is a property of the straining motion associated with the small eddies or mean 
vorticity, it is plausible to assume that $\sigma_{\infty}=\frac{5}{2}$ when $\mu \overline{\mathbf{H}}^{2} \approx \rho(\epsilon v)^{\frac{1}{2}}$, i.e. when there is equipartition of energy between the magnetic field and the small eddies, where $\epsilon$ is the rate at which energy is dissipated by viscosity.

The magnetic spectrum for $k>k_{d}$ is then given by (3.8) with $\sigma_{\infty}=\frac{5}{2}$ and is

$$
\Gamma(k)=\frac{8}{3 \pi^{\frac{1}{2}}} \overline{\mathbf{H}^{2}}\left(\frac{\lambda}{-\gamma}\right)^{\frac{5}{2}} k^{4} e^{\lambda k^{2} / \gamma},
$$

where the constant of proportionality may now be fixed by the requirement that the magnetic energy is almost entirely in components with $k>k_{d}$. Also, the Ohmic dissipation is

$$
D=-\frac{5}{2} \gamma \mu \overline{\mathbf{H}^{2}} \approx \rho \epsilon,
$$

so that there is also rough equality of dissipation by viscosity and electrical resistivity in the dynamo.

The magnetic spectrum is sharply peaked near $k=k_{\epsilon}$ (see figure 1) with the bulk of the magnetic energy in components with wave-number $k_{c}$, and is very different from the cases shown in figures 2 and 3 where the magnetic energy resides mainly in components of wave-number $k_{d}$ or less. The spectrum function in the inertial subrange is still given by (3.7), from which we obtain

$$
\Gamma(k)=\overline{\mathbf{H}}^{2}(\lambda / \nu)^{\frac{5}{2}} k_{d}^{-\frac{4}{3}} k^{\frac{1}{3}} .
$$

It is interesting that the structure of the magnetic field in the dynamo, if it exists, depends upon the magnetic diffusivity however small this may be.

According to the present model of magnetic energy generation and transfer, the inertial subrange eddies cannot by themselves amplify the magnetic field at a rate sufficient to overcome the transfer of energy to larger wave-numbers. In other words, once equipartition at large wave-numbers is set up, a steady state can be maintained and there is no mechanism by which the magnetic energy in components with smaller wave-numbers can grow; unless (3.3) is violated and the vorticity analogy is wrong. This is difficult to accept, and the conclusion must be that if the turbulent dynamo exists, its strength is given by Batchelor's criterion and not by that of Biermann and Schliiter.

To conclude, we return to the case where the dynamo does not exist and speculate briefly about what happens when the energy of the applied field $\frac{1}{2} \mu \overline{H_{0}^{2}} \approx \rho u^{\prime 2}$. According to our model, $\sigma$ is then zero for all wave-numbers large compared with $L^{-1}$ and there is approximate equipartition of energy between components of $\mathbf{u}$ and $\mathbf{H}$ in the inertial subrange. Thus

$$
(\mu \mid \rho) \Gamma(k) \approx E(k) \approx \epsilon^{\frac{2}{3}} k^{-\frac{5}{3}} \quad \text { for } \quad L^{-1} \ll k \ll k_{d} .
$$

For $k>k_{d}, E(k)$ is negligible because of viscous dissipation, but $\Gamma(k)$ continues with a $k^{-1}$ behaviour until $k \approx k_{c}$. The amount of magnetic energy in the spectrum tail is readily found to be of order

$$
\rho u^{\prime 2} R^{-\frac{1}{2}} \log (v / \lambda) \text {. }
$$

Thus if $\log (\nu / \lambda) \gg R^{\frac{1}{2}}$, amplification of the magnetic energy is still possible.

This paper was written during the tenure of a Resident Research Fellowship at the Jet Propulsion Laboratory, California Institute of Technology. 


\section{REFERENCES}

BATCHELOR, G. K. 1950 Proc. Roy. Soc. A, 201, 405.

BATChELOR, G. K. 1959 J. Fluid Mech. 5, 113.

BiermanN, L. \& SChLÜTeR, A. 1950 Z. Naturforsch. $5 a, 237$.

Coresin, S. 1961. J. Fluid Mech. 11, 407.

Eulison, T. H. 1962 Mécanique de la Turbulence, p. 113. Editions du Centre National de la Recherche Scientifique, Paris.

Howells, I. D. 1960 J. Fluid Mech. 9, 104.

MOFFATT, K. $1961 \mathrm{~J}$. Fluid Mech. 11, 625.

SafFMan, P. G. 1963 J. Fluid Mech. 16, 545. 